\title{
The Theory of Branching Processes for a Description of the Configurational Statistics of Branched Polycondensate Polymers
}

\author{
S. V. Korolev, S. I. Kuchanov, and M. G. Slin'Ko \\ Karpov Institute of Physical Chemistry, ul. Obukha 10, \\ 107120 Moscow B-120, USSR
}

(Received December 8, 1982)

\begin{abstract}
This article presents an original algorithm for calculating, using the theory of branching processes, the probabilities of branched macromolecule fragments of different configurations. This multipurpose algorithm is illustrated for the simplest possible case of equilibrium homopolycondensation with due regard for substitution effects of various orders. The number of independent parameters of the kinetic model and experimental methods which may be used for determinating these parameters are discussed.
\end{abstract}

KEY WORDS Branched Polymers / Configurational Statistics / Equilibrium Polycondensation / Network Formation / Branching Processes / Gelation /

The preceding article ${ }^{1}$ formulated a method for describing the configurational statistics of branched polymers by specifying the mean numbers $c\left(U_{k, q}\right)$ of various fragments $\left(k\right.$-ads $\left.\left(U_{k, q}\right)\right)$ of molecules.* These numbers can be calculated using the theory of random branching processes introduced by Gordon in his pioneering paper. ${ }^{2}$ This theory has been found $^{3-7}$ quite applicable to random copolycondensation (both equilibrium and nonequilibrium) and to equilibrium copolycondensation of any monomer mixture involving substitution effects (SE) of the first order, or first-shell substitution effects $\left(\mathrm{FSSE}^{4}\right)$. In the following is proved that this theory is applicable for equilibrium polycondensation with SE of the second order $\left(\mathrm{SSSE}^{8}\right)$. There are grounds to believe that in this way a discription can be made of the products of equilibrium polycondensation with SE of an arbitrary order because in this case, the probability of any molecule is independent of the order in which its links (bonds) are formed. Thus, it may be considered that these links are obtained by monomer attachment in an order associated with some random branching process. ${ }^{9}$ For nonequilibrium polycon- densation, a branching process does not, generally speaking, agree with an FSSE model ${ }^{10}$ but in many cases it can be used as a good approximation.

The proper choice of branching process convenient for calculating the statistical characteristics of a polymer can usually be made in several ways. For instance, its individuals (particles which give birth to other such particles) may be associated with monomer units and functional groups or with intramolecular links. The final results of the calculation do not depend on the choice of process as was shown for random polycondensation and for the FSSE model. ${ }^{3,5.6}$ Individuals of branching process

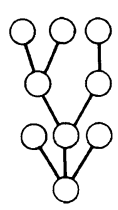

a

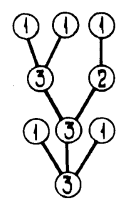

b

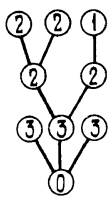

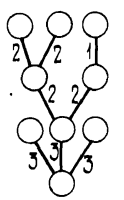

d
Figure 1. Ways to choose correspondence between individuals of a branching process and elements of a rooted ordered tree associated with a molecule.

* The notation used in the preceding article will be preserved and the formulae and figures in it will be labeled with a prime. 
can also be classified into types by coloring. Thus in Figure 1a all the individuals are the same. In Figures $1 \mathrm{~b}$ and $1 \mathrm{c}$ the individuals have colors denoted as $0,1,2$, and 3. Similarly, in Figure 1d the colored individuals are links. An additional division of individuals into types is in some cases necessary to provide more detailed information on the configurational statistics of macromolecules.

\section{PROBABILITIES OF DIRECTED SEQUENCES}

For calculating the number of various molecule fragments the probabilities $P\left\{U_{k, q}\right\}_{r}$ of directed $k$ $\operatorname{ads}^{1}$ are necessary (eq $10^{\prime}$ of ref 1 ). Let us show how $P\left\{U_{k, q}\right\}_{r}$ can be found using the theory of random branching processes. An ordered tree may be regarded as a family tree defined by the family history. ${ }^{11}$ The probability of an ordered tree is equals to the product of probabilities of giving birth to a certain litter by each of the individuals. If there are different types of individuals, that product should be multiplied by the probability of the initial individual being of a specified type. Thus for any branching process with two types of individuals represented as the black and white vertices, the probability of the tree in Figure $2^{\prime}$, can be represented as $p_{0} p_{1} p_{2}^{3} p_{3}^{6}$ where $p_{0}$ is the probability that a black individual is in the zeroth generation, $p_{1}$, the probability that a black root will give birth to three black individuals, and $p_{2}$ the probability of a black individual of the first generation having two white children. Finally, $p_{3}$ is the probability that a white individual of the second generation will have no descendants. To determine $P\left\{U_{k, q}\right\}_{r}$, it is necessary to add up the probabilities of all the trees starting with the $k$-ad $\left\{U_{k, q}\right\}_{r}$. It is easy to see that $P\left\{U_{k, q}\right\}_{r}$ is the product of the above form over all elements of the the $k$-ad $\left\{U_{k, q}\right\}_{r}$.

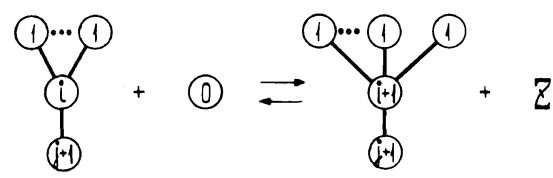

Figure 2. Scheme of a reaction of monomer attachment to a monomer unit of the $i$-th kind linked with $(i-1)$ units of the first kind and a unit of the $(j+1)$-th kind; a by-product $z$ is formed.

\section{FIRST-SHELL SUBSTITUTION EFFECT FOR DISCRIBING THE F-FUNCTIONAL HOMOPOLYCONDENSATION}

In this paper all general ideas are illustrated for the $f$-functional homopolycondensation of the monomer $\mathrm{SA}^{f}$. The homopolymer which then forms can be regarded as a copolymer in which the monomer unit of the $i$-th $(i=0,1, \cdots, f)$ type coincides with the homopolymer unit $S(i)$ of the $i$-th kind (or with the unit in which $i$ of $f$ functional groups have reacted). Any molecule is characterized by a vector $\boldsymbol{l}$ and a matrix $\boldsymbol{B}, l_{i}$ being the number of units $S(i)$, and $b_{i j}=b_{j i}$ is the number of links between units $S(i)$ and $S(j)$ in it. Such links are referred to as links of kind $(i j)$. Isomers enumerated in an arbitrary way by the subscript $q$ are molecules with the same values of $\boldsymbol{l}$ and $\boldsymbol{B}$. The share $f_{W}(\boldsymbol{l}, \boldsymbol{B} ; q)$ of units taken by the $q$-th isomer of the $(\boldsymbol{l}, \boldsymbol{B})$-mer determines the function $f_{W}(\boldsymbol{l}, \boldsymbol{B} ; q)$ of the molecular configurational distribution (MCD) and the sum $f_{W}(\boldsymbol{l}, \boldsymbol{B} ; q)$ over all $q$ 's specifies the function $f_{W}(\boldsymbol{l}, \boldsymbol{B})$ of the size-composition-functionality-distribution (SCFD) because in equilibrium, bonds of various kinds $(i j)$ in the presence of SE should be regarded as internal functional groups of various types.

Let us illustrate a probabilistic measure on directed sequences with an example ${ }^{6}$ of a branching process which describes equilibrium homopolycondensation in an FSSE model. Its individuals are divided into two classes. An individual of the $i$-th type of the first class $(i=0,1, \cdots, f)$ is associated with the monomer unit of the $i$-th kind (in Figure 1b such an individual is represented as a graph vertex of the $i$-th color). Individuals of the second class are all identical (uncolored) and each is associated with a pair of reacted functional groups, or the link which is represented in Figure $1 \mathrm{~b}$ as a graph edge. Probability generating functions (PGF's) $\mathscr{F}_{i}^{(0)}$ and $\mathscr{F}_{i}$ of the number of children of an individual of the first class in the zeroth and in all other generations and the PGF $\mathscr{F}$ for the children of individuals of the second class, respectively, have the form: ${ }^{6}$

$$
\mathscr{F}_{i}^{(0)}(x)=x^{i}, \quad \mathscr{F}_{i}(x)=x^{i-1}, \quad \mathscr{F}(s)=\sum_{j=1}^{f} d_{j} s_{j}
$$

$$
d_{j}=j \lambda_{j} / p f, \quad p f=\sum_{j=1}^{f} j \lambda_{j}
$$


Here $p$ is the conversion of functional groups, $\lambda_{j}$, the fraction of units of the $j$-th kind equal to the probability that the branching process starts with an individual of the $j$-th type and $d_{j}$ is equal to the fraction of reacted groups of units of the $j$-th kind among all reacted groups. From eq 1, it follows that an individual of the $i$-th type gives rise, with a probability of 1 , to exactly $i$ children of the second class if the individual is of the zeroth generation and in all other generations, it gives birth, with the same probability, to $(i-1)$ such children. Consequently, its associated multiplier in the above product (which is equal to the probability of the ordered sequence) is unity. An individual from the second class gives birth to exactly one child of the first class which is found to be of the $j$-th type with a probability of $d_{j}$. Consequently, in this case, it is associated with the multiplier $d_{j}$. Since the probability that an individual of the $i$-th type will appear in the zeroth generation is $\lambda_{i}$, and since the multiplier $d_{j}$ occurs as many times as there are nonroot units $S(j)$ in the $k$-ad, the general algorithm leads to the probability

$$
P\left\{U_{k, q}\right\}_{r}=\lambda_{i} \prod_{j}\left(d_{j}\right)^{k_{j}-\delta_{i j}}
$$

of the $k$-ad $\left\{U_{k, q}\right\}_{r}$ which has $k_{j}$ units of the $j$-th kind $(j=1, \cdots, f)$ and a root of the $i$-th kind. Here $\delta_{i j}$ is the Kronecker delta. Bearing in mind the relation (eq 2) between $\lambda_{j}$ and $d_{j}$, we can see that, as noted in the preceding paper, ${ }^{1}$ the product $i \cdot P\left\{U_{k, q}\right\}_{r}$ of the probability (eq 3 ) of the $k$-ad by the degree $i$ of its root is the same for all $\left\{U_{k, q}\right\}_{r}$ associated with a certain sequence $\left(U_{k, q}\right)$. Furthermore, in the FSSE model, the product is the same for all $k$-ads $\left\{U_{k, q}\right\}_{r}$ having the same value of the vector $\boldsymbol{k}=$ $\left\{k_{1}, k_{2}, \cdots, k_{f}\right\}$.

The number of undirected sequences $\left(U_{k, q}\right)$ is obtained through eq 3 and $10^{\prime}$. Family trees

Table I. Numbers of various dyads $\left(U_{2, q}\right)$ and triads $\left(U_{3, q}\right)$ shown in Figure 1, ref 1

\begin{tabular}{rlllll}
\hline$q$ & $c\left(U_{2, q}\right)$ & $q$ & $c\left(U_{3, q}\right)$ & $q$ & $c\left(U_{3, q}\right)$ \\
\hline 0 & $3 p d_{1}^{2} / 2$ & 0 & $3 p d_{1}^{2} d_{2} / 2$ & 6 & $6 p d_{1} d_{3}^{2}$ \\
1 & $3 p d_{1} d_{2}$ & 1 & $3 p d_{1} d_{2}^{2}$ & 7 & $3 p d_{2}^{2} d_{3}$ \\
2 & $3 p d_{1} d_{3}$ & 2 & $3 p d_{1}^{2} d_{3}$ & 8 & $3 p d_{2}^{2} d_{3}$ \\
3 & $3 p d_{2}^{2} / 2$ & 3 & $3 p d_{1} d_{2} d_{3}$ & 9 & $3 p d_{2} d_{3}^{2} / 2$ \\
4 & $3 p d_{2} d_{3}$ & 4 & $3 p d_{2}^{3} / 2$ & 10 & $6 p d_{2} d_{3}^{2}$ \\
5 & $3 p d_{3}^{2} / 2$ & 5 & $6 p d_{1} d_{2} d_{3}$ & 11 & $3 p d_{3}^{3}$ \\
\hline
\end{tabular}

of the branching process which we describe are $T$-trees ${ }^{1}$ since the individuals associated with unreacted groups are absent. Consequently, the numbers of $D$-trees in the formulae of the preceding paper ${ }^{1}$ should be replaced by corresponding values of $T$ trees. As a result, we have for monads the obvious expression $c\left(U_{1, i}\right)=P\left\{U_{1, i}\right\}=\lambda_{\mathrm{i}}$ and the number of dyads and triads of Figure $1^{\prime}$ is summarized in the Table I.

Applying eq 3 and $6^{\prime}$ to sequences that are $(\boldsymbol{l}, \boldsymbol{B})$-mers and summing up all equivalence classes with the same degree of vertices $i$ and all $(\boldsymbol{l}, \boldsymbol{B})$ mers with the same values of the vector $l$ leads to the fraction $f_{W}(l)$ of units in a molecule obtained by Luby $^{12}$ as

$$
f_{W}(\boldsymbol{l})=\sum_{\boldsymbol{B}, q} f_{W}(\boldsymbol{l}, \boldsymbol{B} ; q)=l T_{(i)}(l) p f \prod_{j}\left(d_{j}\right)^{l_{j}} / i l_{i}
$$

Here $T_{(i)}(\boldsymbol{l})$ is the number of various ordered $T$ trees with $l_{j}$ vertices of degree $j(1 \leqslant j \leqslant f)$ and a root of the degree $i . T_{(i)}(l)$ is equal to, ${ }^{12,13}$

$$
T_{(i)}(l)=\frac{(l-2) !}{l_{1} ! l_{2} ! \cdots l_{f} !} i l_{i}
$$

In the particular case of ideal homopolycondensation

$$
\begin{aligned}
& \lambda_{i}=C_{f}^{i} p^{i}(1-p)^{f-i}, \\
& d_{i}=C_{f-1}^{i-1} p^{i-1}(1-p)^{f-i},
\end{aligned}
$$

and the number $c\left(U_{k, q}\right)$ of sequences $\left(U_{k, q}\right)$ with $k^{\prime}$ external edges obtained through eq 3 and $10^{\prime}$ has a more compact form if $T$-trees (whose probability is computed by eq 3 ) are replaced by $D$-trees using eq $12^{\prime}$ and $7^{\prime}$.

$$
\begin{aligned}
& k c\left(U_{k, q}\right)=D(k, q) p^{n}(1-p)^{m}, \\
& n=k-1+k^{\prime}, \quad m=(f-2) k+2-k^{\prime} .
\end{aligned}
$$

If the sequence $\left(U_{k, q}\right)$ is a $k$-mer, or includes no connecting edges $\left(k^{\prime}=0\right)$, then summing up eq 5 all $k$-mers leads to the well-known expression ${ }^{3}$ for the molecular weight distribution (MWD)

$$
f_{W}(k)=D(k) p^{k-1}(1-p)^{(f-2) k+2}
$$

where $D(k)$ is the total number of various ordered $D$-trees with $k$ nodes of degree $f$. The generating function (GF) of these numbers is easily obtained by the graph theory ${ }^{3,14}$

$$
\sum_{k=1}^{\infty} D(k) s^{k}=\tilde{g}(s)=s(1+\tilde{u})^{f}, \quad \tilde{u}=s(1+\tilde{u})^{f-1}
$$


Note that individuals of the first and second classes of the above branching process consistently alternate. Therefore we can limit ourselves to either class. Thus, in considering individuals only of the second class leads to the well-known ${ }^{4}$ branching process with one type of individuals (Figure 1a). Though in ref 4 they are associated with monomer units, all final formulae remain unchanged if a branching process is used in which the individuals are associated with links. ${ }^{3}$ This branching process with one type of individuals has the simplest form but is less informative on the configurational statistics of the molecules than the initial process specified by eq 1 . Thus, instead of the GF of the distribution $f_{W}(\boldsymbol{l})$ of eq 4 , this branching process leads only to the GF of the MWD (eq 6). If we limit ourselves to individuals of the first class, then the details of the configurational statistics of polymers with the aid of the resultant process do not different from that determined by the initial branching process (1). The PGF's of one individual of the $i$-th type in the zeroth $\mathrm{F}_{i}^{(0)}$ and all other generations $\mathrm{F}_{i}$ have the form ${ }^{6}$

$$
\mathrm{F}_{i}^{(0)}(s)=\left(\sum_{j=1}^{f} d_{j} s_{j}\right)^{i}, \quad \mathrm{~F}_{i}(s)=\left(\sum_{j=1}^{f} d_{j} s_{j}\right)^{i-1}
$$

\section{SECOND-SHELL SUBSTITUTION EFFECT}

Let us now proceed to the design of a branching process in the SSSE model. A similar mathematical description has been suggested earlier ${ }^{8,9}$ but no rigorous proof of its applicability has been available. Reference 9 has established the relation of parameters introduced formally in ref 8 with equilibrium constants of elementary reactions that are the thermodynamic parameters of the system.

Formulae of the branching process will be derived in the same manner as in the case of FSSE in ref 6. Let us first find the bond energy $\Delta F^{*}(\boldsymbol{l}, \boldsymbol{B} ; q)$ of an arbitrary molecule, which is equal to the difference in the energy of the $(\boldsymbol{l}, \boldsymbol{B} ; q)$-isomer and that of $l$ monomers. The change in the standard free energy $\Delta F(\boldsymbol{l}, \boldsymbol{B} ; q)$ of formation of that isomer from monomers is different from $\Delta F^{*}(\boldsymbol{l}, \boldsymbol{B} ; q)$ by the value $T \Delta S(\boldsymbol{l}, \boldsymbol{B} ; q)$ where $T$ is the absolute temperature and $\Delta S$, the change in the combinatorial entropy ${ }^{15}$ resulting from that reaction. The quantities $\Delta F(\boldsymbol{l}, \boldsymbol{B} ; q)$ dictate the equilibrium concentra- tions $c(\boldsymbol{l}, \boldsymbol{B} ; q)$ of molecules. To determine SCFD $f_{W}(\boldsymbol{l}, \boldsymbol{B}), \quad c(\boldsymbol{l}, \boldsymbol{B} ; q)$ should be added up for all isomers $q$ having the same value of $\Delta F^{*}$ and differing in the value of $\Delta S$. Changes in the combinatorial enetropy depend only on the topology of the resultant molecule and can be found as in the case of ideal copolycondensation. ${ }^{7}$ This permits reducing the PGF of SCFD to a standard form in the branching process theory.

In this model, a change in the energy $\Delta F^{*}(l, B ; q)$ is the sum of partial energies $F_{i j}^{*}$ of all its bonds of various ( $i j$ ) kinds whose numbers in the molecule are equal to $b_{i j}$. Since their linear combination

$$
i l_{i}=\sum_{j=1}^{f} b_{i j}\left(1+\delta_{i j}\right)
$$

determines the numbers $l_{i}$ of units of the $i$-th kind in the molecule, another equivalent representation is possible in which the energy of the molecule is associated with its monomer units as well as its bonds. Associating each unit of the $i$-th kind with an energy $F_{i}(i>0)$ and monomer, with $F_{0}=0$, the bounds are associated with energies $F_{i j}<F_{i j}^{*}$; and

$$
\Delta F^{*}(\boldsymbol{l}, \boldsymbol{B} ; q)=\sum_{i \leqslant j} b_{i j} F_{i j}^{*}=\sum_{j=1}^{f} l_{i} F_{i}+\sum_{i \leqslant j} b_{i j} F_{i j}
$$

The number of independent thermodynamic parameters $F_{i j}^{*}$, $i \leqslant j$, of the model is obviously equal to the number $f(f+1) / 2$ of various kinds of links (ij). Consequently, in the second representation, $f$ arbitrary relations should be imposed on the thermodynamic parameters $F_{i}$ and $F_{i j}$ whose number is equal to $f(f+3) / 2$.

To establish eq 9 in strict terms, let us make use of the basic property of equilibrium polycondensation reactions ${ }^{3}$ whereby the energy of formation of any molecule does not depend on the way in which it forms and is equal to the sum of energy increments at each stage. Let this way of formation of the $q$-th isomer of the $(\boldsymbol{l}, \boldsymbol{B})$-mer (which is unambiguously determined by the sequence of formation of links in it) be a sequence of monomer attachments dictated by representation of that isomer as a family tree in Figure 1. Thus, one can enumerate the links in that tree rightwards for all generations starting with the zeroth and assume that they form in this sequence. At each stage the monomer attaches to the unit $S(i)$ of some $i$-th kind whose "children" are all of the first kind and the kind of its "father" is $j+1,0 \leqslant j \leqslant f-1$, in Figure 2. 
If the unit $S(i)$ is in the zeroth generation, then it has no "father" and automatically $j=0$. Change in the energy $\Delta F_{i}(j)$ as a result of that attachment is expressed as an equilibrium constant $k_{i}(j)$ of that elementary reaction which, with $i>0$, can be represented as

$$
k_{i}(j)=\frac{\tilde{\Phi}(i+1)}{\tilde{\Phi}(i)} \frac{\Phi(i, j)}{\Phi(i-1, j)} k_{0}(0), \quad \begin{aligned}
& 1 \leqslant i \leqslant f-1, \\
& 0 \leqslant j \leqslant f-1,
\end{aligned}
$$

using the definition of $\tilde{\Phi}(i), \Phi(i, j)=\Phi(j, i)^{9}$ and some other expressions of ref 9. Consequently, energy change $\Delta F_{i}(j)=-k T \ln k_{\mathrm{i}}(j)$ as a result of one stage can be represented as

$$
\begin{gathered}
\Delta F_{i}(j)=F_{i+1}-F_{i}+F_{1}-F_{0}+F_{i+1, j+1}-F_{i, j+1} \\
\quad+F_{i+1,1}, \quad 1 \leqslant i \leqslant f-1, \quad 0 \leqslant j \leqslant f-1, \\
\Delta F_{0}(0)=-k T \ln k_{0}(0), \\
F_{i}=-k T \ln \tilde{\Phi}(i), \quad F_{i j}=-k T \ln \left(k_{0}(0) \Phi(i-1, j-1)\right), \\
\tilde{\Phi}(0)=\tilde{\Phi}(1)=\Phi(i, 0)=1
\end{gathered}
$$

where $k$ is the Boltzmann constant. Equation 11 can be interpreted in a simple way so that the truth of eq 9 is proved. This follows at once from the stoichiometric scheme of the equilibrium reaction in Figure 2, which involves units and bonds of different kinds:

$$
\begin{aligned}
& S(i)+S(0)+(i, j+1) \stackrel{k_{i}(j)}{\rightleftarrows} \\
& \quad S(i+1)+S(1)+(i+1, j+1)+(i+1,1)+z
\end{aligned}
$$

In addition to the above way of specifying $f(f+1) / 2$ independent thermodynamic parameters $F_{i j}^{*}$, there are other equivalent sets of such parameters. One is characterized ${ }^{9}$ by equilibrium constants $k_{i}(j)$ for $0 \leqslant i \leqslant j \leqslant f-1$, another by parameters $\tilde{\Phi}(i) \quad(2 \leqslant i \leqslant f), \quad \Phi(i, j) \quad(1 \leqslant i \leqslant j \leqslant f-1)$ and $k_{0}(0)$, and another by energies $F_{i}(1 \leqslant i \leqslant f-1)$ and $F_{i j}(1 \leqslant i \leqslant j \leqslant f)$. It has been noted above that $f$ relations can be imposed on the parameters $F_{i}$ and $F_{i j}$. From eq 12, these relations are in this case $F_{11}=F_{21}=\cdots=F_{f 1}, F_{1}=0$. Each of these sets of independent parameters can be obtained from the knowledge of any other. Thus, from eq 8 and 9 with due regard to the above relations on $F_{i}, F_{i j}$ we have

$$
\begin{aligned}
& F_{i j}^{*}=F_{i} / i+F_{j} / j+F_{i j} ; \quad F_{i}=i\left(F_{i 1}^{*}-F_{11}^{*}\right), \\
& F_{i j}=F_{i j}^{*}-F_{i 1}^{*}-F_{j 1}^{*}+2 F_{11}^{*}
\end{aligned}
$$

The remaining sets of parameters are expressed in terms of each other through eq 12 and 10 and the definition of $\tilde{\Phi}(i)$ and $\Phi(i, j) .{ }^{9}$

The concentration $c(\boldsymbol{l}, \boldsymbol{B} ; q)$ of specific $(\boldsymbol{l}, \boldsymbol{B} ; q)$ isomers is found through the law of mass action for the polymolecular reaction of its formation from $l$ monomers

$$
\frac{c(\boldsymbol{l}, \boldsymbol{B} ; q) z^{l-1}}{M^{l}}=\exp \left(-\frac{\Delta F(\boldsymbol{l}, \boldsymbol{B} ; q)}{k T}\right)
$$

where $z$ and $M=\lambda_{0}$ are the concentrations (as $c(\boldsymbol{l}, \boldsymbol{B} ; q)$ is, per monomer unit) of the condensation by-product and the monomer, respectively. 'The change $\Delta F$ in free energy as a result of this reaction is dependent on $\Delta F^{*}$ of eq 9 and change in the combinatorial entropy $\Delta S^{15,7}$

$$
\begin{aligned}
& \Delta F(\boldsymbol{l}, \boldsymbol{B} ; q)=\Delta F^{*}(\boldsymbol{l}, \boldsymbol{B} ; q)-T \Delta S(\boldsymbol{l}, \boldsymbol{B} ; q), \\
& \Delta S(\boldsymbol{l}, \boldsymbol{B} ; q)=k \ln \left(\left(\mathscr{S}_{D}(1)\right)^{l} / \mathscr{S}_{D}(\boldsymbol{l}, \boldsymbol{B} ; q)\right)
\end{aligned}
$$

where $\mathscr{S}_{D}(1)=f !$ and the order $\mathscr{S}_{D}(\boldsymbol{l}, \boldsymbol{B} ; q)$ of the automorphism group of the $D$-tree which represents the $(\boldsymbol{l}, \boldsymbol{B} ; q)$-isomer can be expressed in terms of an analogous quantity $\mathscr{S}_{T}(\boldsymbol{l}, \boldsymbol{B} ; q)$ for the corresponding $T$-tree

$$
\mathscr{S}_{D}(l, \boldsymbol{B} ; q)=\mathscr{S}_{T}(\boldsymbol{l}, \boldsymbol{B} ; q) \prod_{i}((f-i) !)^{l_{i}}
$$

since each automorphim of the $T$-tree is associated with a set of automorphisms of the $D$-tree which are different in permutations of $(f-i)$ unreacted groups in each unit of the $i$-th kind. From eq 9 and 12-15 we have

$$
\begin{aligned}
c(\boldsymbol{l}, \boldsymbol{B} ; q)= & \prod_{i=1}^{f}\left(\frac{f ! \tilde{\Phi}(i) \lambda_{0}}{(f-i) !}\right)^{l_{i}} \\
& \times \prod_{i \leqslant j}\left(\frac{k_{0}(0)}{z} \Phi(i-1, j-1)\right)^{b_{i j}} \frac{1}{\mathscr{S}_{T}(\boldsymbol{l}, \boldsymbol{B} ; q)}
\end{aligned}
$$

Application of eq $7^{\prime}$ to $T$-trees enables summation of eq 16 all $q$ 's and finding the GF $G(s, X)$ of the $\operatorname{SCFD} f_{W}(\boldsymbol{l}, \boldsymbol{B})$

$$
\begin{aligned}
G(\boldsymbol{s}, \boldsymbol{X}) & =\sum_{\boldsymbol{l}, \boldsymbol{B}} f_{W}(\boldsymbol{l}, \boldsymbol{B}) \prod_{i}\left(s_{i}\right)^{l_{i}} \prod_{i \leqslant j}\left(x_{i j}\right)^{b_{i j}} \\
= & \sum_{\boldsymbol{l}, \boldsymbol{B}}\left(\sum_{i} \frac{T_{(i)}(\boldsymbol{l}, \boldsymbol{B})}{i}\right) \prod_{i}\left(\frac{f ! \tilde{\Phi}(i) \lambda_{0} s_{i}}{(i-1) !(f-i) !}\right)^{l_{i}} \\
& \times \prod_{i \leqslant j}\left(\frac{k_{0}(0)}{z} \Phi(i-1, j-1) x_{i j}\right)^{b_{i j}}
\end{aligned}
$$


where $T_{(i)}(\boldsymbol{l}, \boldsymbol{B})$ is the number of various ordered $T$ trees with a root of the $i$-th kind associated with all $(\boldsymbol{l}, \boldsymbol{B})$-mers. Changing the order of summation in this formula we have the expression

$$
\begin{aligned}
G(s, X) & =\sum_{i=1}^{f} \tilde{g}_{i}\left(s^{\prime}, X^{\prime}\right) / i, \quad s_{i}^{\prime}=i C_{f}^{i} \tilde{\Phi}(i) \lambda_{0} s_{i}, \\
x_{i j}^{\prime} & =\frac{k_{0}(0)}{z} \Phi(i-1, j-1) x_{i j}
\end{aligned}
$$

of the function $G$ through GF's $\tilde{g}_{i}$ of the number $T_{(i)}(\boldsymbol{l}, \boldsymbol{B})$ that can be obtained using the algorithm of ref 14 .

$$
\tilde{g}_{i}\left(s^{\prime}, \boldsymbol{X}^{\prime}\right)=s_{i}^{\prime}\left(\sum_{j=1}^{f} x_{i j}^{\prime} \tilde{u}_{j}\right)^{i}, \quad \tilde{u}_{i}=s_{i}^{\prime}\left(\sum_{j=1}^{f} x_{i j}^{\prime} \tilde{u}_{j}\right)^{i-1}
$$

Consequently, the GF $G$ is determined by eq 17 and 18 in terms of the parameters $\tilde{\Phi}(i), \Phi(i, j)$, $\lambda_{0}$, and $k_{0}(0) / z$. To redüce $G$ to a form standard in the theory of branching processes, let us express $k_{0}(0) / z$ in terms of auxiliary variables $\omega_{i}, i=1, \cdots$, $f$, using the relations of ref 9 .

$$
\begin{aligned}
k_{0}(0) / z & =\omega_{i} / f \Sigma_{i} \lambda_{0}, \\
\Sigma_{i} & =\sum_{j=1}^{f} C_{f-1}^{j-1} \tilde{\Phi}(j) \Phi(i-1, j-1)\left(\omega_{j}\right)^{j-1}, \\
\lambda_{0} & =\left(\sum_{j=0}^{f} C_{f}^{j} \tilde{\Phi}(j)\left(\omega_{j}\right)^{j}\right)^{-1} .
\end{aligned}
$$

Following the replacement

$$
\tilde{u}_{i}\left(s^{\prime}, X^{\prime}\right)=f C_{f-1}^{i-1}\left(\omega_{i}\right)^{i-1} u_{i}(s, X)
$$

with due regard to eq 19 , the GF can be represented as

$$
\begin{aligned}
G(s, \mathbf{1}) & =\sum_{i=0}^{f} \lambda_{i} s_{i} \mathrm{~F}_{i}^{(0)}(\boldsymbol{u}), \quad u_{i}=s_{i} \mathrm{~F}_{i}(\boldsymbol{u}), \\
\mathrm{F}_{i}^{(0)}(s) & =\left(\sum_{j=1}^{f} d_{j}(i) s_{j}\right)^{i}, \quad \mathrm{~F}_{i}(s)=\left(\sum_{j=1}^{f} d_{j}(i) s_{j}\right)^{i-1}, \\
\lambda_{i} & =C_{f}^{i} \tilde{\Phi}(i)\left(\omega_{i}\right)^{i} \lambda_{0}, \\
d_{j}(i) & =C_{f-1}^{j-1} \tilde{\Phi}(j) \Phi(i-1, j-1)\left(\omega_{j}\right)^{j-1} / \Sigma_{i}
\end{aligned}
$$

Here we assume that $x_{i j}=1$ for all elements of the matrix $X=1$. Note that, with fixed thermodynamic parameters $\tilde{\Phi}(i), \Phi(i, j)$ and $k_{0}(0) / z$, the value of $k_{0}(0) / z$ and eq 19 unambiguously determine the vector $\omega=\left\{\omega_{1}, \cdots, \omega_{f}\right\}$ and consequently the values of $\lambda_{i}$ and $d_{j}(i)$ and also the conversion $p$ expressed in terms of $\lambda_{i}$ in eq 2 . Consequently, the parameters $\lambda_{i}$ and $d_{j}(i)$ of the branching process implicitly depend, through the vector $\omega$, on the conversion $p$ and on the above thermodynamic parameters.

Equation 20 has a standard form in the theory of branching processes. Equation 20 has the following sense. With a probability of $\lambda_{i}$ the branching process, as in the FSSE model, starts with an individual of the $i$-th type associated with a monomer unit of the $i$-th kind (Figure 1b). The PCFs $\mathrm{F}_{i}^{(0)}$ and $\mathrm{F}_{i}$ for the children of one such individual in the zeroth and all other generations are straightforward generalizations of the PGF's (eq 7) and $\mathrm{F}_{i}^{(0)}$ and $F_{i}$ (eq 20) become eq 7 in the absence of SSSE when $\Phi(i, j)=1$ for all $i, j$. The dependence of $d_{j}(i)$ on $i$ is thus equivalent to SSSE when the reproductive behaviour of some individual depends on the kind of the unit which is associated with the father of the individual. As in the case of ideal polycondensation, ${ }^{4}$ the PGF's of the zeroth and all other generations are related as

$$
\mathrm{F}_{i}(s)=\sum_{j=1}^{f} \frac{\partial \mathrm{F}_{i}^{(0)}(s)}{\partial s_{j}} / \sum_{j=1}^{f} \frac{\partial \mathrm{F}_{i}^{(0)}(\mathbf{1})}{\partial s_{j}}
$$

The earlier ${ }^{8,9}$ branching processes for the SSSE model are different from the above one with respect to the correspondence between individuals and graph elements. Thus the branching process of ref 8 always starts, with an individual (associated with a unit) of the special type 0 found only in the zeroth generation. Individuals of the subsequent generations belong, by definition, to the $i$-th type if they have $(i-1)$ "brothers" (Figure 1c). The PGF's $\tilde{\mathrm{F}}_{i}^{(l)}$ for the children of one such individual in the $l$-th generation can be expressed in terms of the parameters of eq 21 in the form

$$
\begin{aligned}
& \tilde{\mathrm{F}}_{i}^{(0)}(s)=\sum_{i=0}^{f} \lambda_{i}\left(s_{i}\right)^{i}, \quad \tilde{\mathrm{F}}_{i}^{(1)}(s)=\sum_{j=0}^{f-1} d_{j+1}(i)\left(s_{j}\right)^{j}, \\
& \hat{\mathrm{F}}_{i}^{(l)}(s)=\sum_{j=0}^{f-1} d_{j+1}(i+1)\left(s_{j}\right)^{j}, \quad l \geqslant 2
\end{aligned}
$$

In ref 9 the individuals were links whose $i$-th type is also dictated by the number $(i-1)$ of their "brothers" ( $c f$. Figures 1d and c). The PGF's for the children of one such particle have the same form (eq 22) as in ref 8 . The parameters of such a proc- 
ess introduced in ref 9 are expressed in a simple way in terms of $\lambda_{i}$ and $d_{j}(i)$ of eq 21: $p^{(0)}(i)=\lambda_{i}$ and $p_{i}^{(1)}(j)=d_{j+1}(i)$ while their relation with the parameters introduced in ref 8 is given in ref 9 . The probabilistic measure on the trees and, consequently, all final results naturally coincide for all three branching processes.

\section{Selection of Parameters of the Model and Deter- mination of Adequacy}

The probabilistic parameters (eq 21) of the above branching process are related in a simple way with the experimental numbers of various dyads consisting of two units, $S(i)$ and $S(j)$. These numbers coincide with the concentrations $Q_{i-1, j-1}$ of links of the kind (ij) per monomer unit. Choosing the unit $S(i)$ as the root of the directed dyad $\{i j\}$ let us find its probability from the theory of branching processes

$$
P\{i j\}=\lambda_{i} d_{j}(i)
$$

where $\lambda_{i}$ is the probability that the branching process starts with an individual of the $i$-th type and $d_{j}(i)$, the probability that its child is of the $j$-th type. Since there are $i$ ordered $T$-dyads $\{i j\}$ the concentration $Q_{i-1, j-1}$ is, according to eq $10^{\prime}$, equal to the product of the probability formula (eq 23) by $i$ that should be halved with $i=j$ when both vertices of the dyad belong to the same equivalence class. Applying the same reasoning to the directed dyad $\{j i\}$ with a root $S(j)$ yields the formula

$$
Q_{i-1, j-1}\left(1+\delta_{i j}\right)=i \lambda_{i} d_{j}(i)=j \lambda_{j} d_{i}(j),
$$

which, together with normalization conditions or with their equivalent stoichiometric relations

$$
\sum_{j=1}^{f} d_{j}(i)=1, \quad i \lambda_{i}=\sum_{j=1}^{f} Q_{i-1, j-1}\left(1+\delta_{i j}\right)
$$

determines all parameters of the branching process $\lambda_{i}$ and

$$
d_{j}(i)=Q_{i-1, j-1}\left(1+\delta_{i j}\right) / \sum_{s=1}^{f} Q_{i-1, s-1}\left(1+\delta_{i s}\right)
$$

in terms of link concentrations $Q_{i, j}$.

In an actual determination of concentrations $Q_{i, j}$, for instance by NMR spectroscopy, ratios of these concentrations rather than these quantities $Q_{i, j}$ are determined. This enables obtaining the values of $d_{j}(i)$ from eq 26 but the fractions $\lambda_{i}, i=1, \cdots$, $f$, are found from the eq 25 only up to an un-

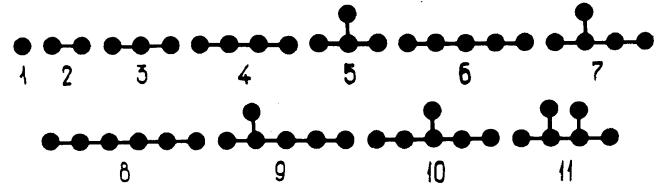

Figure 3. A set of low molecular oligomers sufficient for determining thermodynamic parameters in an SSSE model of equilibrium homopolycondensation of a threefunctional monomer.

specified factor. To find this factor, the fraction $\lambda_{i}$ of some monad $S(i)$ should be also measured and normalized together with others $\lambda_{0}+\lambda_{1}+\cdots+\lambda_{f}=$ 1 so as to determine that factor and values of all $\lambda_{i}$, $0 \leqslant i \leqslant f$. Note that the number of independent probabilistic parameters, according to eq 24 26 , is equal to the number $f(f+1) / 2$ of possible kinds of links (ij) and to the number of the above independent thermodynamic parameters of eq 9-12. Thus, specifying $f(f+1) / 2$ quantities $d_{i}(j), 1 \leqslant i<j \leqslant f$ and $\lambda_{i}, 1 \leqslant i \leqslant f$ permits finding $\lambda_{0}=1-\lambda_{1}-\cdots-\lambda_{f}, d_{i}(j)$ with $i>j$ from the second equality (eq 24) and $d_{i}(i)$, from the conditions (eq 25) of normalizing the quantities $d_{i}(j)$.

There is still another way to determine the parameters of our model through measurement of relative concentrations of the most low molecular weight reaction products such as those of Figure 3 by chromatographic methods for instance. Once the concentrations $c_{i}$ of oligomers numbered as $i$ (Figure 3) have been calculated by the above theoretical relations, the independent thermodynamic parameters can be expressed through experimental values of $c_{i}$. Thus, with the polycondensation of a three-functional monomer to determine the parameters $k_{i}(j), i \geqslant j$, it is sufficient to measure the relative concentrations of the by-product $z$ and molecules enumerated as $1-5,7$, and 11

$$
\begin{aligned}
k_{0}(0)=\frac{2}{9} \frac{z}{c_{1}} \frac{c_{2}}{c_{1}}, k_{1}(0) & =\frac{1}{6} \frac{z}{c_{1}} \frac{c_{3}}{c_{2}}, k_{2}(0)=\frac{z}{c_{1}} \frac{c_{5}}{c_{3}}, \\
k_{1}(1)=\frac{1}{6} \frac{z}{c_{1}} \frac{c_{4}}{c_{3}}, k_{2}(1) & =\frac{1}{3} \frac{z}{c_{1}} \frac{c_{7}}{c_{4}}, \\
k_{2}(2) & =\frac{4}{3} \frac{z}{c_{1}} \frac{c_{11}}{c_{7}} .
\end{aligned}
$$

Note that six independent constant $k_{\mathrm{i}}(j)$ are determined through seven ratios of concentrations $c_{i} / c_{j}$ and $z / c_{1}$. In the case of a closed system, the latter quantity is not independent and can be ex- 
pressed in terms of the others since $z / c_{1}$ is essentially calculated through constants $k_{i}(j)$. In addition to $k_{i}(j)$, there is one more independent parameter for an open system, the concentration of the withdrawn by-product, $z_{w}=f p / 2-z$. The probabilistic parameters $\lambda_{i}$ and $d_{j}(i)$ can be found either in a similar way or by calculating through $k_{i}(j)$ and $z$ or $p$, using the relations (eq 19 and 21) and the dependences of the parameters $\tilde{\Phi}(i)$ and $\Phi(i, j)$ on $k_{i}(j) .{ }^{9}$ To verify the adequacy of the chosen SSSE model for a specific chemical system, it should be determined whether the experimental values of oligomer concentrations $c_{i}$ satisfy certain theoretical relations, such as

$$
\begin{aligned}
& \frac{c_{8}}{c_{6}}=\frac{c_{6}}{c_{4}}=6 \frac{c_{1}}{z} k_{1}(1)=\frac{c_{4}}{c_{3}}, \quad \frac{c_{9}}{c_{6}}=3 \frac{c_{1}}{z} k_{2}(1)=\frac{c_{7}}{c_{4}}, \\
& \frac{c_{10}}{c_{6}}=3 \frac{c_{1}}{z} \frac{k_{2}^{2}(1)}{k_{2}(0)}=\frac{1}{3} \frac{c_{3} c_{7}^{2}}{c_{4}^{2} c_{5}}
\end{aligned}
$$

Another way to check the adequacy is to determine the probabilistic parameters of the branching processes, calculate the numbers $c\left(U_{k}\right)$ of various $k$-ads or concentrations $c(\boldsymbol{l}, \boldsymbol{B} ; q)$ of certain isomers, and compare these with experimental data. For the theoretical calculation of these quantities, it is necessary to find the probabilities $P\left\{U_{k, q}\right\}_{r}$ of associated directed $k$-ads.

\section{Probabilities of Directed $k$-Ads}

The probabilities $P\left\{U_{k, q}\right\}_{r}$ in this model are found in the following way. Let $i_{0}$ denote the kind of unit which is the root of the $k$-ad $\left\{U_{k, q}\right\}_{r}$. With the direction "from the root" links of various kinds also become directed links. Transition from a unit of the $i$-th kind to one of the $j$-th kind is a directed link $\{i j\} \neq\{j i\}$. If the $k$-ad $\left\{U_{k, q}\right\}_{r}$ has $\beta_{i j}$ such links $\{i j\}$, then its probability is

$$
P\left\{U_{k, q}\right\}_{r}=\lambda_{i_{0}} \prod_{i, j}\left(d_{j}(i)\right)^{\beta_{i j}}
$$

In addition to the degree $i_{0}$ of the root, this probability depends only on the numbers $b_{i j}, k_{i}, k_{i}^{\prime}$ of the undirected links $(i j)$, units $S(i)$, and connecting links ${ }^{1}$ which issue from such units in the $k$-ad $\left\{U_{k, q}\right\}_{r}$ because, in compliance with (24) and (29),

$$
\begin{aligned}
P\left\{U_{k, q}\right\}_{r} & =\frac{1}{i_{0}} \prod_{i \leqslant j}\left(Q_{i-1, j-1}\left(1+\delta_{i j}\right)\right)^{b_{i j}} \prod_{i}\left(i \lambda_{i}\right)^{-\kappa_{i}} \\
\kappa_{i} & =(i-1) k_{i}-k_{i}^{\prime}
\end{aligned}
$$

Thus the probability of the tree of Figure 1 is

$$
\begin{aligned}
P\left\{U_{9}\right\} & =\lambda_{3} d_{1}(2)\left(d_{1}(3)\right)^{4} d_{2}(3)\left(d_{3}(3)\right)^{2} \\
& =\frac{Q_{0,1}\left(Q_{0,2}\right)^{4}\left(2 Q_{2,2}\right)^{2}}{3 \cdot 2 \lambda_{2}\left(3 \lambda_{3}\right)^{6}}
\end{aligned}
$$

The probability of the directed trail $\left\{i_{0} i_{1} \cdots i_{n}\right\}$, or of a linear sequence of units of the kinds $i_{0}, i_{1}, \cdots, i_{n}$ $\left(i_{k} \neq 1\right.$ with $\left.0<k<n\right)$ has, in compliance with eq 29 , the form

$$
P\left\{i_{0} i_{1} \cdots i_{n}\right\}=\lambda_{\mathrm{i}_{0}} d_{i_{1}}\left(i_{0}\right) d_{i_{2}}\left(i_{1}\right) \cdots d_{i_{n}}\left(i_{n-1}\right)
$$

which almost coincides with the probability of the realization $\left\{i_{0}, i_{1}, \cdots, i_{n}\right\}$ of a Markov chain with states $1,2, \cdots, f$ and a transition matrix $\boldsymbol{D}$ with elements $d_{j}(i)$. The difference is in that instead of components $\pi_{i}$ of the fixed probability vector $\pi$ for $D$, eq 31 includes quantities $\lambda_{i}$ determined by the matrix $\boldsymbol{D}$ up to an unspecified factor through an equation obtained from eq 24 and 25 and is akin to the equation for determining the vector $\pi$

$$
i \lambda_{i}=\sum_{j} j \lambda_{j} d_{j}(i), \quad \pi_{i}=\sum_{j} \pi_{j} d_{j}(i)
$$

To find an uncertain factor, either $\lambda_{0}$ or $p$ should be additionally specified and then either the normalizing condition on $\lambda_{i}$ or the second equality of eq 2 should be used.

\section{Numbers of Trails, Gel-Point, and Sol-Fraction}

The numbers $c\left(U_{k, q}\right)$ of the sequences $\left(U_{k, q}\right)$ are determined from the probabilities $P\left\{U_{k, q}\right\}_{r}$ by eq $10^{\prime}$. For instance, permutation of branches in the tree of Figure 1 leads to six different ordered trees and its root is one of two elements of its equivalence class; therefore the number of associated isomers is $c\left(U_{9}\right)=3 P\left\{U_{9}\right\}$. In the case of linear sequences, or trails $\left\{i_{0} i_{1} \cdots i_{n}\right\}$, the number of associated ordered trees is easy to find. Let us choose the extreme unit $S\left(i_{0}\right)$ as the root and then change, in all possible ways, the position of the unique child of each individual $S\left(i_{0}\right), S\left(i_{1}\right), \cdots$, $S\left(i_{n-1}\right)$ among its connecting edges. For the root we have $i_{0}$ such positions, for the individuals $S\left(i_{k}\right)$, $0<k<n$ we have $i_{k}-1$ positions. The last individual $S\left(i_{n}\right)$ has no children and so all its identical connecting edges are traced in a unique way. Consequently, the number of different directed $T$-trails $\left\{i_{0} i_{1}{ }^{*} \cdots i_{n}\right\}$ is equal to

$$
T_{i_{0}}\left(i_{0} i_{1} \cdots i_{n}\right)=i_{0}\left(i_{1}-1\right) \cdots\left(i_{n-1}-1\right)
$$


Bearing in mind eq 29 and $10^{\prime}$ we find the number of undirected trails

$$
\begin{aligned}
& c\left(i_{0} i_{1} \cdots i_{n}\right) \\
& \quad=i_{0} \lambda_{i_{0}} \prod_{k=1}^{n-1}\left(\left(i_{k}-1\right) d_{i_{k}}\left(i_{k-1}\right)\right) d_{i_{n}}\left(i_{n-1}\right) / \sigma\left(i_{0} \cdots i_{n}\right)
\end{aligned}
$$

where $\sigma\left(i_{0} \cdots i_{n}\right)$ is equal to 2 for symmetrical and 1 for the remaining trails. Thus, the number of various triads that can be regarded as trails (ijk) is obtained from eq 32 with due regard for eq 26 and 25

$$
c(i j k)\left(1+\delta_{i k}\right)=\frac{j-1}{j \lambda_{j}} Q_{i-1, j-1}\left(1+\delta_{i j}\right) Q_{j-1, k-1}\left(1+\delta_{j k}\right)
$$

Equation 31 holds, naturally, also for the FSSE where the probabilities $d_{j}(i)$ are independent of $i$. This formula immediately leads to the number of various dyads and triads if the relation of $\lambda_{j}$ and $d_{j}$ in eq 2 is used (see the Table I). The numbers of trails in the case of ideal copolycondensation are reported in ref 7 .

With the numbers $c\left(i_{0} \cdots i_{n}\right)$ of all trails available and a certain conformational model of the chain assumed, one can calculate various physicochemical properties of the polymer following the lines of ref 16. Note, however, that for a model of ideal polycondensation, the number of trails of different lengths $n$ was found ${ }^{16}$ belonging to $l$-mers with a certain $l$ while eq 32 provides less detailed information in the sense that only the total number of trails can be determined belonging to all molecules (with all values of $l=1,2, \cdots$ ). Consequently, this formula can lead only to a few of the characteristics calculated in ref 16 such as only $z$-average rather than number-average or weightaverage radius of gyration. Equation 32, however, is derived for SSSE and makes possible more realistic conformational models where conformational probability of the chain depends on the kinds $(i j)$ of links. Note that in earlier papers, this dependence was neglected.

A condition for the gel-point is that the determinant of the matrix with elements

$$
\delta_{i j}-\frac{\partial F_{i}(\mathbf{1})}{\partial s_{j}}=\delta_{i j}-(i-1) d_{j}(i)
$$

vanishes. After the gel-point all the above findings can be used for calculating the configurational statistics of the sol-fraction. For this purpose, modified PGFs ${ }^{17}$ should be used which are obtained in the following way. First it is necessary to find the components $u_{i}^{*}<1$ of the vector $u^{*}$ by solving the equations $u_{i}^{*}=\mathrm{F}_{i}\left(\boldsymbol{u}^{*}\right)$ with functions $\mathrm{F}_{i}$ determined in eq 20 . Then modified values of the parameters $\hat{\lambda}_{i}$ and $\hat{d}_{j}(i)$ should be found through eq 21 where $\omega_{i}$ should be replaced by $\hat{\omega}_{i}=\omega_{i}\left(u_{i}^{*}\right)^{1 /(i-1)}$. Modified PGFs are obtained from eq 20 by replacing the parameters $\lambda_{i}$ and $d_{j}(i)$ by $\hat{\lambda}_{i}$ and $\hat{d}_{j}(i)$ with $\hat{\lambda}_{i}$ equal to fractions of units of the $i$-th kind among all units of the sol-fraction. The share of units taken by the solfraction is equal to

$$
\omega_{\mathrm{sol}}=\sum_{i=0}^{f} \lambda_{i} \mathrm{~F}_{i}^{(0)}\left(\boldsymbol{u}^{*}\right)
$$

\section{REMARKS ON SUBSTITUTION EFFECTS OF HIGHER ORDERS}

The results of this paper suggest possible extensions of the theory to SE models of higher orders. In the case of an SE of the third order, for instance, the individuals of the branching process can be characterized by a pair of numbers $(i j)$ which denote the kinds of units associated with the individual and its "father." In a model of the SE of the fourth order, the individual type depends also both on the kinds of units that represent its "grandfather" and "brothers," etc. The probabilistic parameters of the branching processes should be expressed as the numbers $c\left(U_{k, q}\right)$ of various $k$-ads of an increasing diameter. Note that the number of individual types rapidly increases with the SE order; even more so does the number of probabilistic parameters of the branching process which should be found experimentally.

\section{THE CHOICE OF MODEL USING EXPERIMENTAL DATA}

In conclusion, let us formulate the algorithm for the choice of a kinetic model for specific equilibrium polycondensation systems provided that experimental data are available on the number of sequences $\left(U_{k, q}\right)$ with small values of $k$. In order to find whether the system is ideal, it is sufficient to confine oneself to an experimental determination of the fractions $\lambda_{i}$ of any two monads; for convenience one of these can be a monomer. For random polycon- 
densation system, the following condition should be met:

$$
\lambda_{i}=C_{f}^{i}\left(\lambda_{0}\right)^{1-\mathrm{i} / f}\left(1-\lambda_{0}^{1 / f}\right)^{i}
$$

Otherwise the SE should be recognized in the design of a kinetic model. Furthermore, to be able to use only an FSSE model, all the ratios

$$
\begin{array}{r}
Q_{i-1, j-1}\left(1+\delta_{i j}\right) / Q_{i-1, k-1}\left(1+\delta_{i k}\right)=j \lambda_{j} / k \lambda_{k}, \\
1 \leqslant j, k \leqslant f
\end{array}
$$

of the concentrations $Q_{i-1, j-1}$ of various links should be independent of the first subscript $i$. If one of these conditions is not met, similar relations between triads should be verified:

$$
\begin{aligned}
c(i j k)\left(1+\delta_{i k}\right) / c(i j l)\left(1+\delta_{i l}\right) & \\
=Q_{j-1, k-1}\left(1+\delta_{j k}\right) / Q_{j-1, l-1}\left(1+\delta_{j l}\right), & 1 \leqslant j, k, l \leqslant f
\end{aligned}
$$

where there is obviously no dependence on the first subscript $i$. Feasibility of eq 35 is the criterion for applicability of an SSSE model. The further sequence of actions in this algorithm is obvious.

A kindred algorithm for the choice of a kinetic model can be used if data on concentrations of lower molecular oligomers are available. Thus, with polycondensation of a three-functional monomer the criteria (eq 33-35) can be replaced, for instance, by

$$
\begin{aligned}
& c_{2}=\frac{3}{2} c_{1}^{4 / 3}\left(1-c_{1}^{1 / 3}\right), \quad c_{3}=3 c_{1}^{5 / 3}\left(1-c_{1}^{1 / 3}\right)^{2}, \\
& c_{4}=7 c_{1}^{2}\left(1-c_{1}^{1 / 3}\right)^{3} ; \\
& \frac{c_{3}}{c_{2}}=\frac{c_{4}}{c_{3}}=\frac{c_{7}}{3 c_{5}}, \quad \frac{c_{11}}{c_{7}}=\frac{c_{7}}{4 c_{3}}=\frac{c_{10}}{4 c_{6}},
\end{aligned}
$$

and eq 28 where $c_{i}$ are concentrations of molecules numbered as $i$ in Figure 3 .

For nonequilibrium polycondensation, the possibility of describing the configurational statistics of its products in terms of a branching process does not generally follow from the kinetic FSSE model. ${ }^{10}$ This is especially true of SE models of higher orders. There are grounds to believe, however, that as Markov chains of higher order are increasingly accurate in describing the distribution of units in non-Markov linear copolymers, ${ }^{18}$ so the higher the level in the above hierarchical sequence of branching processes, the better the approxi- mation of SE models by such a process. Concentrations of monads, dyads, etc. which determine, by eq 2 and 26 , the probabilistic parameters $d_{j}$ and $d_{j}(i)$ of branching processes eq 7 and 20 are calculated in this case by solving kinetic equations for $c\left(U_{k, q}\right)$. What term of the hierarchical sequence should be chosen depends on the accuracy of experimental methods for determining the number of sequences or oligomer concentrations.

The algorithm suggested above for equilibrium polycondensation systems makes it also possible to use the relations. (eq 33-37 and 28) to determine which of the branching processes of the hierarchical sequence describes the nonequilibrium system with an accuracy sufficient for practical purposes. Its parameters are calculated by using eq 2 and 26 where experimental values of monads, dyads, etc. are substituted.

The above mentioned principles were used for describing configurational statistics of ureaformaldehyde resins. The content of different molecule fragments was determined using ${ }^{13} \mathrm{C}$ NMR spectra. ${ }^{19}$ A part of these data were used for determination of probabilistic parameters for the branching process. The remaining experimental meanings of fragment fractions were compared with theoretical ones and the latter were calculated using the theory of branching processes. According to this comparison, a conclusion on the possibility of the use of a given modification of branching process was made. It was found that the simplest branching process satisfactorily describing the studied samples corresponds to the first-shell substitution effect model for formaldehyde and third-shell substitution effect for urea. The results of the study of configurational statistics of urea-formaldehyde resins will be published in a separate paper.

\section{REFERENCES}

1. S. I. Kuchanov, S. V. Korolev, and M. G. Slin'ko, Polym. J., 15, 775 (1983).

2. M. Gordon, Proc. R. Soc. London, Ser. A, 268, 240 (1962).

3. S. I. Kuchanov, "Metody Kineticheskikh Raschetov v Khimii Polimerov" ("Methods of Kinetic Calculations in the Chemistry of Polymers"), Khimiya, Moscow, 1978.

4. M. Gordon and G. R. Scantlebury, Trans. Faraday Soc., 60, 604 (1964).

5. S. V. Korolev, S. I. Kuchanov, and M. G. Slin'ko, Dokl. Akad. Nauk USSR, 258, 1157 (1981). 
6. S. V. Korolev, S. I. Kuchanov, and M. G. Slin'ko, Dokl. Akad. Nauk USSR, 262, 1422 (1982).

7. S. V. Korolev, S. I. Kuchanov, and M. G. Slin'ko, Dokl. Akad. Nauk USSR, 263, 633 (1982).

8. M. Gordon and T. G. Parker, Proc. Roy. Soc. (Edinburg), Ser. A, 69, 181 (1971).

9. Ye. B. Brun and S. I. Kuchanov, Vysokomol. Soedin., Ser. A, 21, 1393 (1979).

10. S. I. Kuchanov, Dokl. Akad. Nauk USSR, 249, 899 (1979).

11. T. E. Harris, "The Theory of Branching Processes," Springer Verlag, 1963.

12. P. Luby, J. Phys. Chem., 78, 1083 (1974).

13. M. Gordon and J. A. Torkington, Discrete Appl.
Math., 2, 207 (1980).

14. I. J. Good, Proc. Cambridge Phil. Soc., 61, 499 (1965).

15. M. Gordon and W. B. Temple, J. Chem. Soc. A, 729 (1970).

16. K. Kajiwara, J. Chem. Phys., 54, 296 (1971).

17. G. R. Dobson and M. Gordon, J. Chem. Phys., 41, 2389 (1964).

18. N. A. Plate, A. D. Litmanovich, and O. V. Noa, "Makromolekularnye Reaktsii" ("Macromolecular Reactions"), Khimiya, Moscow, 1977.

19. I. Ya. Slonim, et al., Vysokomol. Soedin., Ser. A, 19, 776 (1977). 